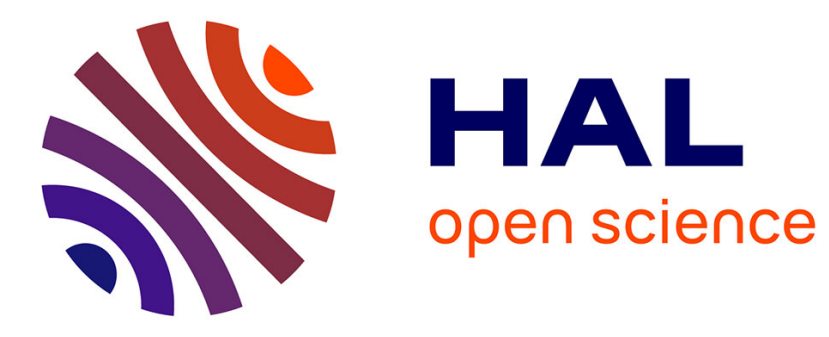

\title{
Purely Cubic Spin Splittings with Persistent Spin Textures
}

Hong Jian Zhao, Hiro Nakamura, Rémi Arras, Charles Paillard, Peng Chen, Julien Gosteau, Xu Li, Yurong Yang, Laurent Bellaiche

\section{- To cite this version:}

Hong Jian Zhao, Hiro Nakamura, Rémi Arras, Charles Paillard, Peng Chen, et al.. Purely Cubic Spin Splittings with Persistent Spin Textures. Physical Review Letters, 2020, 125 (21), 10.1103/PhysRevLett.125.216405 . hal-03295043

\section{HAL Id: hal-03295043 \\ https://hal.science/hal-03295043}

Submitted on 28 Oct 2021

HAL is a multi-disciplinary open access archive for the deposit and dissemination of scientific research documents, whether they are published or not. The documents may come from teaching and research institutions in France or abroad, or from public or private research centers.
L'archive ouverte pluridisciplinaire HAL, est destinée au dépôt et à la diffusion de documents scientifiques de niveau recherche, publiés ou non, émanant des établissements d'enseignement et de recherche français ou étrangers, des laboratoires publics ou privés. 


\title{
Purely Cubic Spin Splittings with Persistent Spin Textures
}

\author{
Hong Jian Zhao $\odot,{ }^{1,2, *}$ Hiro Nakamura, ${ }^{1}$ Rémi Arras, ${ }^{3}$ Charles Paillard $\odot,{ }^{4,1,2}$ Peng Chen $\odot,{ }^{1,2, \dagger}$ Julien Gosteau, ${ }^{3}$ \\ $\mathrm{Xu} \mathrm{Li},{ }^{5}$ Yurong Yang ${ }^{5}{ }^{5}$ and Laurent Bellaiche ${ }^{1,2, \$}$ \\ ${ }^{1}$ Physics Department, University of Arkansas, Fayetteville, Arkansas 72701, USA \\ ${ }^{2}$ Institute for Nanoscience and Engineering, University of Arkansas, Fayetteville, Arkansas 72701, USA \\ ${ }^{3}$ CEMES, Université de Toulouse, CNRS, UPS, 29 Rue Jeanne Marvig, F-31055 Toulouse, France \\ ${ }^{4}$ Laboratoire SPMS, CentraleSuplec/CNRS UMR8580, Université Paris-Saclay, 8-10 Rue Joliot-Curie, 91190 Gif-sur-Yvette, France \\ ${ }^{5}$ Jiangsu Key Laboratory of Artificial Functional Materials, Nanjing University, Nanjing 210093, China
}

(Received 11 June 2020; revised 10 August 2020; accepted 9 October 2020; published 20 November 2020)

\begin{abstract}
Purely cubic spin splittings in the band structure of bulk insulators have not been extensively investigated yet despite the fact that they may pave the way for novel spin-orbitronic applications and can also result in a variety of promising spin phenomena. By symmetry analysis and first-principles simulations, we report symmetry-enforced purely cubic spin splittings (SEPCSS) that can even lead to persistent spin textures. In particular, these SEPCSS can be thought to be complementary to the cubic Rashba and cubic Dresselhaus types of spin splittings. Strikingly, the presently discovered SEPCSS are expected to exist in the large family of materials crystallizing in the $\overline{6} m 2$ and $\overline{6}$ point groups, including the $\mathrm{Ge}_{3} \mathrm{~Pb}_{5} \mathrm{O}_{11}, \mathrm{~Pb}_{7} \mathrm{Br}_{2} \mathrm{~F}_{12}$, and $\mathrm{Pb}_{7} \mathrm{Cl}_{2} \mathrm{~F}_{12}$ compounds.
\end{abstract}

DOI: 10.1103/PhysRevLett.125.216405

Introduction.-The discovery of spin splittings and of the associated spin textures guides a new way toward the spin-orbitronic applications of materials without inversion symmetry, and is attracting considerable attention [1-21]. Most of the discovered spin splittings are consistent with the linear Rashba (LR) or linear Dresselhaus (LD) types, or consist of a mixture between LR and LD kinds [6-9, $11-18,21]$. For the linear spin splittings, the energy levels are dispersed with crystal momentum $(k)$ following $E_{k}=\alpha k^{2} \pm \tau k$, where the $\alpha$ and $\tau$ terms are the effective mass and linear spin splitting terms, respectively. Among the linear spin splittings, one important and promising case is the so-called persistent spin textures (PST) arising when the LR and LD contributions compensate each other, whose spin configurations are $k$ independent, therefore enabling long-range spin transport without dissipation $[7,9,15$, 19-23]; interestingly, the linear-spin-splitting-based persistent spin textures can be well protected by symmetry and thus are not easily destroyed [7]. Moreover, the cubic Rashba (CR) and cubic Dresselhaus (CD) spin splittings, with the band dispersion characterized by $E_{k}=\alpha k^{2} \pm \tau^{\prime} k^{3}$, have started to gain attention recently, possibly due to some unique benefits to spin transport [3-5,24-27]. For example, some important experimental discoveries reported $\mathrm{CR}$ in, e.g., (001) $\mathrm{SrTiO}_{3}$ surface [4], Ge/SiGe quantum well [3], $\mathrm{SrTiO}_{3}$-based heterostructures [28], and surface states of antiferromagnet $\mathrm{TbRh}_{2} \mathrm{Si}_{2}$ [29]. As it will be shown later, the CR effect is usually, by symmetry, mixed with linear in $k$ spin-splitting contributions (note that by mixing we mean that both effects simultaneously exist); consequently, the so far observed CR are mostly based on nonbulk materials (e.g., quantum well, surface, interface) [3,4,28,29]. Focusing on the emerging field of cubic spin splittings, several questions are naturally raised. (i) Are there bulk materials (instead of quantum wells or surfaces or interfaces) presenting symmetry-enforced purely cubic spin splittings (SEPCSS) without mixing with linear in $k$ terms? (ii) Are there SEPCSS (if any) whose mechanisms are beyond $\mathrm{CR}$ and $\mathrm{CD}$ ? (iii) Is it possible to obtain symmetryenforced PST but in the regime of purely cubic spin splittings? Answering these questions may not only enhance the opportunity for original device applications of spin splittings but also enrich the fascinating field of spin-orbit interactions. In particular, addressing (iii) may enable the device applications based on dissipationless spin transport (due to PST) via possible benefits of SEPCSS. Novel purely cubic spin-orbit interaction may also provide a test bed to explore e.g., spin transport in a system with higher spin (e.g., spin 1 and $3 / 2$ quasiparticles) $[19,27,30,31]$. Note that question (i) has already been successfully addressed in zinc-blende-type semiconductors (see, e.g., Refs. [5,32]), some of whose bands are purely $\mathrm{CD}$ type [2]. However, questions (ii) and (iii) remain completely unknown, to the best of our knowledge.

Our main results are as follows. By first deriving the symmetry-allowed lowest order in $k$ spin splittings (twoband model) along all 32 point groups, we do identify some novel SEPCSS, which differ from those associated CR and CD. They occur in $\overline{6}$ and $\overline{6} m 2$ point groups. We further show that these SEPCSS showcase symmetry-enforced persistent spin textures within purely cubic spin splittings. Finally, we propose a family of materials calling for the 
experimental verification of our theory as well as the design of spintronic devices based on SEPCSS with PST.

Results and discussion.-The supporting discussion (Sects. I, II, III) and methods (Sect. IV) are detailed in the Supplemental Material (SM) [33].

Symmetry analysis of the two-band Hamiltonian.-Let us recall that our target is to find possible candidates with SEPCSS, limiting ourselves within the two-band effective Hamiltonian [32] incorporating only the spin degree of freedom (i.e., the degeneracy of orbital degree of freedom, albeit important in some cases [56], is not considered). Our strategy is to derive the possible symmetry-allowed lowest order in $k$ spin-splitting terms, case by case by exhausting all the 32 crystallographic point groups. Note that, by symmetry, only 21 point groups can present such spin splittings (see Sec. I of SM and Table S1 [33]). Out of these 21 , there are 18 point groups allowing linear spin splittings; the other three cases, which are $\overline{6} m 2, \overline{6}$, and $\overline{4} 3 m$, present cubic spin splittings as lowest orders within the framework of the two-band model. Note that none of the terms given in Table S1 [33] correspond to the recently reported cubic Rashba spin-splitting term [i.e., $i\left(k_{x}-i k_{y}\right)^{3}\left(\sigma_{x}+i \sigma_{y}\right)-i\left(k_{x}+i k_{y}\right)^{3}\left(\sigma_{x}-i \sigma_{y}\right)$, with $\sigma_{x}$, $\sigma_{y}$, and $\sigma_{z}$ being Pauli matrices] [3,4]. In other words, from the viewpoint of symmetry, the cubic Rashba must be mixed with linear spin splittings; even though the linear term might be tiny or negligible (see, e.g., Refs. [3,4]), it is allowed by symmetry once the cubic Rashba appears. Also, the cubic spin splittings for $\overline{4} 3 m$ are exactly the cubic Dresselhaus term [2,5].

Typical spin splittings and textures.-In Figs. 1(a)-1(c), we show sketches of band structures for purely linear and cubic spin splittings as well as a mixture of the two. One can clearly see the difference between the purely linear and purely cubic spin splittings; that is, the latter presents zero value of $\partial E_{k} / \partial k$ at $k=0$. At small $k$, the feature of the mixed case is similar to that of the purely linear spin splitting [see Figs. 1(a) and 1(b)]. The spin textures of linear Rashba and Dresselhaus effects [Figs. 1(d) and 1(e)] as well as those of cubic Rashba and Dresselhaus effects [Figs. 1(g) and 1(h)] are also depicted (Fig. 1). Interestingly, Figs. 1(d) and 1(e) show magnetic vortex and antivortex patterns [62] but in reciprocal space instead of the usual real space. The persistent spin texture whose spin configuration is independent of momentum $(k)$ is sketched too for the linear spin-splitting case [Fig. 1(f)]. Strikingly, the spin splittings $\left[k_{y}\left(3 k_{x}^{2}-k_{y}^{2}\right) \sigma_{z}\right.$ and $\left.k_{x}\left(k_{x}^{2}-3 k_{y}^{2}\right) \sigma_{z}\right]$ occurring in $\overline{6} m 2$ and $\overline{6}$ point groups highlight a novel persistent type of spin textures (with cubic in $k$ terms as lowest orders); as we will see below, our proposed PST are "protected" by symmetry and thus are rather robust. In Fig. 1(i), we show the spin texture of $k_{y}\left(3 k_{x}^{2}-k_{y}^{2}\right) \sigma_{z}$ as an example. Such novel spin textures and purely cubic in $k$ spin splittings in $\overline{6} m 2$ and $\overline{6}$ point groups, which are complementary to purely cubic Rashba and
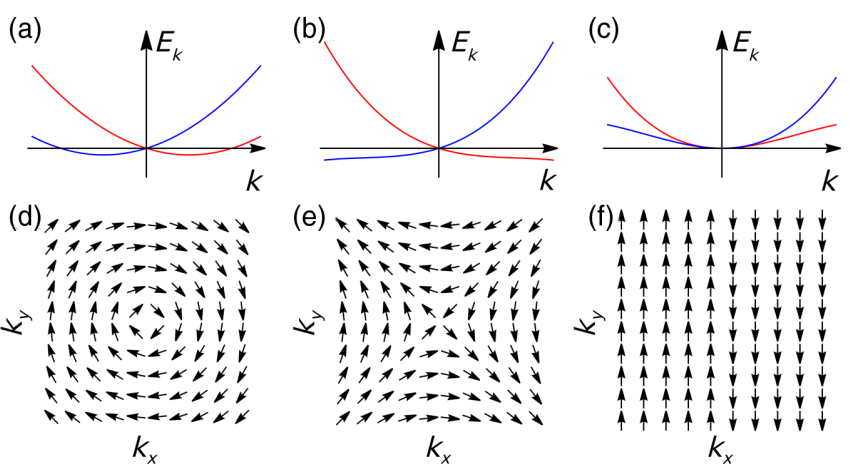

(g) $+1+1 \rightarrow+\infty+1+2$

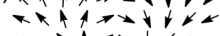
$\leftrightarrow x+\pi v<+4$ $<<+1,1<+\infty$

$x+15 \times 1=19$ $\rightarrow \rightarrow \rightarrow+x \rightarrow \rightarrow$ $\rightarrow \rightarrow 1+2<x \rightarrow b$ $14+2+4 i v$ $k_{x}$

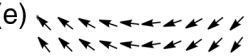

$1+x+2+2<r$ $1+x-2+r$

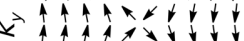

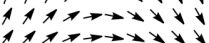

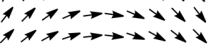

$k_{x}$

(h)

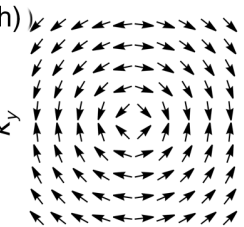

$k_{x}$
$1,1 \rightarrow \infty x+y$ (f) $\uparrow \uparrow \uparrow \uparrow \uparrow \uparrow \downarrow \downarrow \downarrow \downarrow$

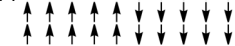

$1 \uparrow \uparrow+\downarrow \downarrow$

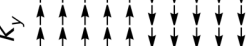

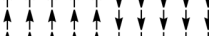

A $k_{x}$

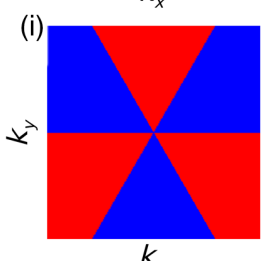

$k_{x}$
FIG. 1. Sketches of the band structures and spin textures due to linear or cubic spin splittings. Panels (a)-(c) depict band energies $E_{k}$ versus $k$ in the form of $E_{k}=\alpha k^{2} \pm \lambda k, E_{k}=\alpha k^{2} \pm \lambda k \pm \eta k^{3}$, and $E_{k}=\alpha k^{2} \pm \eta k^{3}$, respectively. Panels (d) and (e) are schematic spin textures in, e.g., the $m m 2$ point group for linear Rashba (i.e., $-k_{y} \sigma_{x}+k_{x} \sigma_{y}$ ) [1,7] and Dresselhaus (i.e., $k_{y} \sigma_{x}+k_{x} \sigma_{y}$ ) [2,7] spin splittings, respectively. Panel (f) sketches the linear persistent spin textures (e.g., obtained by $k_{x} \sigma_{y}$ ) [7] whose spin configurations are independent of the momentum $k$. Panels $(\mathrm{g})$ and $(\mathrm{h})$ are sketches of spin textures due to cubic Rashba [i.e., $\left.\left(k_{x}-i k_{y}\right)^{3}\left(\sigma_{x}+i \sigma_{y}\right)-\left(k_{x}+i k_{y}\right)^{3}\left(\sigma_{x}-i \sigma_{y}\right)\right][3,4]$ and Dresselhaus [i.e., $\left(k_{x} k_{z}^{2}-k_{x} k_{y}^{2}\right) \sigma_{x}+\left(k_{y} k_{x}^{2}-k_{y} k_{z}^{2}\right) \sigma_{y}+$ $\left(k_{z} k_{y}^{2}-k_{z} k_{x}^{2}\right) \sigma_{z}$ ] [2,5] spin splittings, respectively. Panel (i) shows the persistent spin texture due to cubic spin splittings [e.g., $k_{y}\left(3 k_{x}^{2}-k_{y}^{2}\right) \sigma_{z}$ ]. The spin textures are considered within the $\left(k_{x}, k_{y}\right)$ plane; the arrows and color denote the in-plane and outof-plane components of spin textures, respectively. In panels (d)(h), the spin configurations have in-plane components $s_{x}$ and $s_{y}$ with hardly any out-of-plane components $s_{z}$, while in panel (i) the spin textures merely have $s_{z}$ with the blue (red) colored sectors denoting $s_{z}$ of $-0.5(+0.5)$. In our model, there should be two spin- texture branches associated to two bands; only one branch is shown here for each case.

Dresselhaus types, have seldom been noticed previously. More precisely, we are aware of previous works using $k_{y}\left(3 k_{x}^{2}-k_{y}^{2}\right) \sigma_{z}$ or $k_{x}\left(k_{x}^{2}-3 k_{y}^{2}\right) \sigma_{z}$ to interpret the spin splittings and/or textures in materials with other point groups (e.g., $3 m, 3$; see Refs. $[18,60])$, but the $k_{y}\left(3 k_{x}^{2}-\right.$ $\left.k_{y}^{2}\right) \sigma_{z}$ and/or $k_{x}\left(k_{x}^{2}-3 k_{y}^{2}\right) \sigma_{z}$ terms are often mixed with linear in $k$ spin splittings-and therefore destroy the persistent spin textures (see, e.g., the case for $\mathrm{LaWN}_{3}$ [18]) and is beyond the purely cubic spin splittings.

Route toward the PST with cubic spin splittings. - Let us start from our two-band Hamiltonian for the $\overline{6}$ point group within the $\left(k_{x}, k_{y}\right)$ plane, given by

$$
\begin{aligned}
H\left(k_{x}, k_{y}\right)= & E_{0}+\alpha\left(k_{x}^{2}+k_{y}^{2}\right)+\zeta k_{x}\left(k_{x}^{2}-3 k_{y}^{2}\right) \sigma_{z} \\
& +\lambda k_{y}\left(3 k_{x}^{2}-k_{y}^{2}\right) \sigma_{z},
\end{aligned}
$$


where $\sigma_{z}$ is a Pauli matrix or operator, $\alpha$ is a coefficient related to the effective mass, and $\zeta$ and $\lambda$ characterize spin splitting terms. The eigenvalues of Eq. (1) are easily obtained as $E_{ \pm}=E_{0}+\alpha\left(k_{x}^{2}+k_{y}^{2}\right) \pm \zeta k_{x}^{3} \pm 3 \lambda k_{x}^{2} k_{y} \mp 3 \zeta k_{x} k_{y}^{2} \mp \lambda k_{y}^{3}$. The eigenvectors corresponding to $E_{+}$and $E_{-}$are $|\uparrow\rangle$ and $|\downarrow\rangle$, respectively, which are the eigenstates of the Pauli $\sigma_{z}$ matrix or operator. Our model [Eq. (1)] thus implies that the spin components $\left(s_{x}=s_{y}=0\right.$ and $\left.s_{z}= \pm \frac{1}{2}\right)$ are independent of the momentum $k$, suggesting the persistent spin textures for $\overline{6}$ point groups. A similar conclusion can easily be realized for the $\overline{6} m 2$ case.

Possible candidates. - We now move on to propose the possible candidates that can achieve our abovementioned PST with SEPCSS. In particular, we are interested in the materials $\mathrm{Ge}_{3} \mathrm{~Pb}_{5} \mathrm{O}_{11}, \mathrm{~Pb}_{7} \mathrm{Br}_{2} \mathrm{~F}_{12}$, and $\mathrm{Pb}_{7} \mathrm{Cl}_{2} \mathrm{~F}_{12}$, which were experimentally reported to have the space group of $P \overline{6}$ ( $\overline{6}$ as the associated point group) under appropriate synthesis condition [40,63-65]. We take these materials as our test bed to perform a sequence of simulations (see Sect. IV of the SM [33]) based on density functional theory (DFT). The relaxed crystal structures for these three compounds are shown in Table S2 of the SM [33]. Our crystal structures from DFT coincide with the experimental values; for example, the deviations of the calculated lattice parameters $(a, c)$ from experimental ones [63-65] are $(-0.6 \%,-0.3 \%),(-0.9 \%,-1.5 \%)$, and $(-0.6 \%,-1.1 \%)$, for $\mathrm{Ge}_{3} \mathrm{~Pb}_{5} \mathrm{O}_{11}, \mathrm{~Pb}_{7} \mathrm{Br}_{2} \mathrm{~F}_{12}$, and $\mathrm{Pb}_{7} \mathrm{Cl}_{2} \mathrm{~F}_{12}$, respectively.

The band gaps are about 2.1, 3.6, and $3.8 \mathrm{eV}$ for $\mathrm{Ge}_{3} \mathrm{~Pb}_{5} \mathrm{O}_{11}, \mathrm{~Pb}_{7} \mathrm{Br}_{2} \mathrm{~F}_{12}$, and $\mathrm{Pb}_{7} \mathrm{Cl}_{2} \mathrm{~F}_{12}$, respectively (see Figs. S2 and S3 in SM [33]). Meanwhile, we found that the conduction band minimum (CBM) for $\mathrm{Ge}_{3} \mathrm{~Pb}_{5} \mathrm{O}_{11}$ locates at the $\Gamma$ point, while the $\mathrm{CBM}$ for $\mathrm{Pb}_{7} \mathrm{Br}_{2} \mathrm{~F}_{12}$ is at a valley along the $L-M$ line and that for $\mathrm{Pb}_{7} \mathrm{Cl}_{2} \mathrm{~F}_{12}$ is in the vicinity of the $A$ point (Figs. S2 and S3 of SM [33]). The valley along the $\Gamma-A$ line for $\mathrm{Pb}_{7} \mathrm{Br}_{2} \mathrm{~F}_{12}$ is very close, in terms of energy, to that along the $L-M$ line. In the present work, we will concentrate on the band structures and spin textures around the $\Gamma$ point. Note that even if the $\Gamma$ point for $\mathrm{Pb}_{7} \mathrm{Br}_{2} \mathrm{~F}_{12}$ and $\mathrm{Pb}_{7} \mathrm{Cl}_{2} \mathrm{~F}_{12}$ is not the $k$ point indexing their $\mathrm{CBMs}$, it is still possible in experiments to access the conduction level around the $\Gamma$ point [66].

We compute the band structures from DFT along both the $k_{x}$ and $k_{y}$ directions around the $\Gamma$ point for $\mathrm{Ge}_{3} \mathrm{~Pb}_{5} \mathrm{O}_{11}$, $\mathrm{Pb}_{7} \mathrm{Br}_{2} \mathrm{~F}_{12}$, and $\mathrm{Pb}_{7} \mathrm{Cl}_{2} \mathrm{~F}_{12}$, then we fit those band structures to our model in Eq. (1); the results are shown in Figs. 2(a) and 2(b) in the main text and Fig. S4 in SM [33]. Our model fits the bands very well with the coefficients reported in Table I. Interestingly, the lower conduction bands around the $\Gamma$ point showcase the feature of electrons for $\mathrm{Ge}_{3} \mathrm{~Pb}_{5} \mathrm{O}_{11}$ (i.e., with positive effective mass) and the feature of holes for $\mathrm{Pb}_{7} \mathrm{Br}_{2} \mathrm{~F}_{12}$ and $\mathrm{Pb}_{7} \mathrm{Cl}_{2} \mathrm{~F}_{12}$ (i.e., with negative effective mass), respectively.

Finally, the spin textures ( $\eta$ component $s_{\eta}, \eta=x, y, z$ ) around the $\Gamma$ point within the $\left(k_{x}, k_{y}\right)$ plane are obtained from DFT and also from our parametrized models.
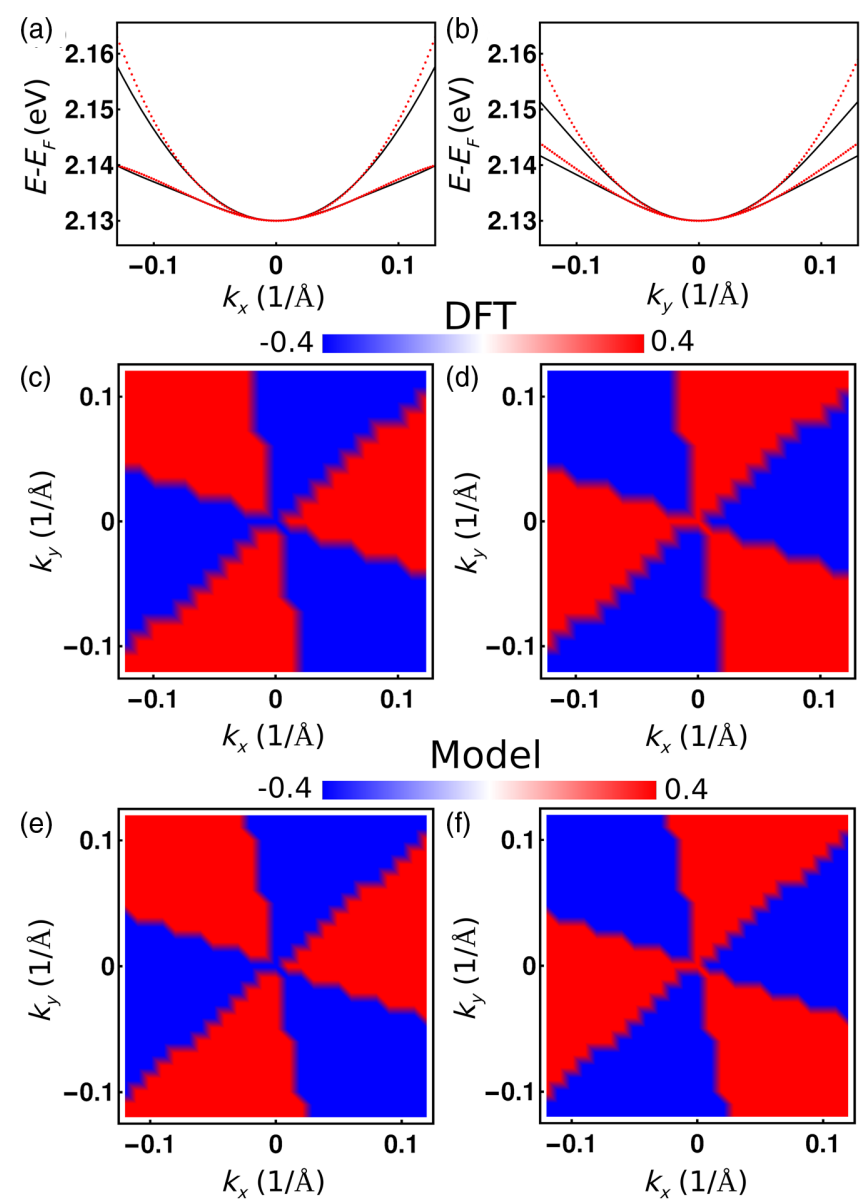

FIG. 2. Band structures of $\mathrm{Ge}_{3} \mathrm{~Pb}_{5} \mathrm{O}_{11}$ around the $\Gamma$ point, along $k_{x}$ (a) and $k_{y}$ (b) directions. The black solid lines and red dots are the band structures obtained by DFT and the model described by Eq. (1), respectively. Panels (c) and (d) are the spin textures calculated by DFT of the outer (lower) and inner (higher) conduction subbands, respectively; panels (e) and (f) are the equivalent of panels (c) and (d) but computed by the model. Only the out-of-plane components ( $s_{z}$, denoted by red and blue colors) are nonzero. Note that the "zigzag" patterns of the border lines separating the positive and negative $s_{z}$ sectors are due to the discrete (coarse) $k$-point sampling (see Sect. IV of the SM [33]). A dense enough $k$-point mesh should result in straight border lines as shown in Fig. 1(i). To reproduce the DFT-based spin textures, we used a similar coarse $k$-point mesh when evaluating the spin textures based on our models.

Figures 2(c)-2(f) are for $\mathrm{Ge}_{3} \mathrm{~Pb}_{5} \mathrm{O}_{11}$, while Figs. S5(a)$\mathrm{S} 5$ (h) (in SM [33]) are for $\mathrm{Pb}_{7} \mathrm{Br}_{2} \mathrm{~F}_{12}$ and $\mathrm{Pb}_{7} \mathrm{Cl}_{2} \mathrm{~F}_{12}$. For all the three investigated compounds, the spin textures obtained by DFT and our models agree with each other. Specifically, our models give $s_{x}=0, s_{y}=0$, and $s_{z}=$ \pm 0.5 for $\mathrm{Ge}_{3} \mathrm{~Pb}_{5} \mathrm{O}_{11}, \mathrm{~Pb}_{7} \mathrm{Br}_{2} \mathrm{~F}_{12}$, and $\mathrm{Pb}_{7} \mathrm{Cl}_{2} \mathrm{~F}_{12}$; in comparison, the DFT-resulted spin textures yield $s_{x}=0$, $s_{y}=0$, and $s_{z}= \pm s_{0}$, with $s_{0}$ ranging from 0.44 to 0.48 for these compounds. This, together with the good fit of the DFT band structures by our models, confirms the validity of our above analysis about the effective Hamiltonian, in 
TABLE I. The parametrization of $E_{0}, \alpha, \beta, \zeta$, and $\lambda$ in our model [Eq. (1)] for the low conduction bands (around the $\Gamma$ point) of $\mathrm{Ge}_{3} \mathrm{~Pb}_{5} \mathrm{O}_{11}, \mathrm{~Pb}_{7} \mathrm{Br}_{2} \mathrm{~F}_{12}$, and $\mathrm{Pb}_{7} \mathrm{Cl}_{2} \mathrm{~F}_{12}$. The $k_{x}$ and $k_{y}$ ranges for fitting our models are $0.188,0.115$, and $0.115 \AA^{-1}$ for $\mathrm{Ge}_{3} \mathrm{~Pb}_{5} \mathrm{O}_{11}, \mathrm{~Pb}_{7} \mathrm{Br}_{2} \mathrm{~F}_{12}$, and $\mathrm{Pb}_{7} \mathrm{Cl}_{2} \mathrm{~F}_{12}$, respectively.

\begin{tabular}{cccc}
\hline \hline & $\mathrm{Ge}_{3} \mathrm{~Pb}_{5} \mathrm{O}_{11}$ & $\mathrm{~Pb}_{7} \mathrm{Br}_{2} \mathrm{~F}_{12}$ & $\mathrm{~Pb}_{7} \mathrm{Cl}_{2} \mathrm{~F}_{12}$ \\
\hline$E_{0}(\mathrm{eV})$ & 2.13 & 3.76 & 3.98 \\
$\alpha\left(\mathrm{eV} \mathrm{\AA}^{2}\right)$ & 1.27 & -1.11 & -2.08 \\
$\zeta\left(\mathrm{eV} \mathrm{\AA}^{3}\right)$ & -5.24 & -2.92 & 1.75 \\
$\lambda\left(\mathrm{eV} \mathrm{\AA}^{3}\right)$ & -3.43 & -5.04 & 8.65 \\
\hline \hline
\end{tabular}

order to describe the SEPCSS with PST for the $\overline{6}$ point group. We also tested our models for the $\overline{6} m 2$ point group. More precisely, we investigated the conduction levels around the $\Gamma$ point of GaSe $(P \overline{6} m 2$ space group) and $\mathrm{K}_{2} \mathrm{HfF}_{6}$ ( $P \overline{6} 2 m$ space group). We found that the spin splittings and textures of these materials are basically well described by our models giving PST as well —with merely some slight in-plane components (see Figs. S6 and S7 and the discussion in the SM [33]).

Furthermore, it is interesting that the spin textures (see Fig. 2 in the main text and Fig. S5 of the SM [33]) for $\mathrm{Ge}_{3} \mathrm{~Pb}_{5} \mathrm{O}_{11}, \mathrm{~Pb}_{7} \mathrm{Br}_{2} \mathrm{~F}_{12}$, and $\mathrm{Pb}_{7} \mathrm{Cl}_{2} \mathrm{~F}_{12}$ are different from each other in the sense that the border lines separating the $+\left|s_{z}\right|$ and $-\left|s_{z}\right|$ sectors vary case by case. Let us elucidate such a fact analytically. Based on the eigenvalues of Eq. (1) we evaluate $k_{y} / k_{x}$ (according to $E_{+}=E_{-}$and assuming that $k_{x}>0$ ) as

$$
\frac{k_{y}}{k_{x}}=\left\{\begin{array}{l}
-\frac{\zeta}{\lambda}-\frac{\left(\zeta^{2}+\lambda^{2}\right)^{2 / 3}}{\lambda(\zeta+i|\lambda|)^{1 / 3}}-\frac{\left(\zeta^{2}+\lambda^{2}\right)^{1 / 3}(\zeta+i|\lambda|)^{1 / 3}}{\lambda} \\
-\frac{\zeta}{\lambda}+\frac{(1-i \sqrt{3})\left[\left(\zeta^{2}+\lambda^{2}\right)^{1 / 3}(\zeta+i|\lambda|)^{1 / 3}\right]}{2 \lambda}+\frac{(1+i \sqrt{3})\left(\zeta^{2}+\lambda^{2}\right)}{2 \lambda\left(\zeta^{2}+\lambda^{2}\right)^{1 / 3}(\zeta+i|\lambda|)^{1 / 3}} \\
-\frac{\zeta}{\lambda}+\frac{(1+i \sqrt{3})\left[\left(\zeta^{2}+\lambda^{2}\right)^{1 / 3}(\zeta+i|\lambda|)^{1 / 3}\right]}{2 \lambda}+\frac{(1-i \sqrt{3})\left(\zeta^{2}+\lambda^{2}\right)}{2 \lambda\left(\zeta^{2}+\lambda^{2}\right)^{1 / 3}(\zeta+i|\lambda|)^{1 / 3}}
\end{array}\right.
$$

In fact, Eq. (2) provides the slope (i.e., $k_{y} / k_{x}$ ) of the three border lines separating the different sectors of the spin textures [note again that the border lines should be straight lines if we use dense enough $k$-point mesh; see Fig. 1(i)].

Summary and perspective.-We have identified purely cubic spin splittings, i.e., $\zeta k_{x}\left(k_{x}^{2}-3 k_{y}^{2}\right) \sigma_{z}+\lambda k_{y}\left(3 k_{x}^{2}-k_{y}^{2}\right) \sigma_{z}$ without any mixing with linear spin splittings, in the nonpolar $\overline{6}$ point group. Such spin splittings are complementary to the cubic Rashba and cubic Dresselhaus types and form robust persistent spin textures which are tunable by varying the ratio between $\zeta$ and $\lambda$ via, e.g., strain engineering, or alloying of different compounds. We further propose three compounds, e.g., $\mathrm{Ge}_{3} \mathrm{~Pb}_{5} \mathrm{O}_{11}$, $\mathrm{Pb}_{7} \mathrm{Br}_{2} \mathrm{~F}_{12}$, and $\mathrm{Pb}_{7} \mathrm{Cl}_{2} \mathrm{~F}_{12}$, to corroborate our theory and achieve our abovementioned effects. Furthermore, we also listed several nonmagnetic and nonmetallic candidates, extracted from the Materials Project [40,41], with $\overline{6}$ and $\overline{6} m 2$ point groups in Table S3 of the SM [33]. This shows that there is a rather big family of materials that can fulfill the PST with SEPCSS; note that all the materials listed in Table S3 (see the SM [33] have been experimentally prepared and they have a wide spectrum of band gaps, thus allowing experimental and technological access to our proposed PST with SEPCSS in $\overline{6}$ and $\overline{6} m 2$ point groups. All in all, our discoveries extend the physics of spin orbits and may also lead to novel nondissipative device application because of these unique cubic spin splittings and persistent spin textures. Also interesting is that materials with symmetry (e.g., $(6 / m) m m$ ) higher than $\overline{6}$ and $\overline{6} m 2$ may still present $\overline{6}$ or $\overline{6} m 2$ symmetry when they are grown as a monolayer (see, e.g., Ref. [67]), or when studying their specific high-symmetric $k$ points whose little group is $\overline{6}$ or $\overline{6} m 2$-as evidenced in, e.g., Ref. [68]. In particular, we point out that purely cubic persistent spin texture we identified in bulk oxides and fluorides in this study may have a natural link to monolayer transition metal dichalcogenides (TMDCs), where out-of-plane spin polarization is also found in a related point group $(\overline{6} \mathrm{~m} 2)$ albeit around the $K$ point of the Brillouin zone [67]. Given the outstanding optoelectronic properties and valley physics found in monolayer TMDCs, further exploration of transport and optical properties in systems having pure cubic interaction could be highly useful [69-71].

Note that our suggested candidates are made of lead and are thus possibly toxic. Hence, discovering lead-free materials presenting large SEPCSS with PST constitutes an interesting avenue to pursue. In particular, a highthroughput screening of corresponding candidates will be highly desired both experimentally and theoretically. A possible starting point may be the lead-free systems listed in Table S3 of the SM [33]. Note also that widely used crystal structure predictors (such as USPEX [72] and CALYPSO [73]) can allow the design of these lead-free materials even from scratch, by, e.g., considering binary and ternary alloys involving heavy elements (implying large spin-orbit coupling).

H. J.Z. and L. B. are thankful for the support from the Department of Energy, Office of Basic Energy Sciences, under Award No. DESC0002220 (H. J. Z. did symmetry analysis, first-principles calculations, and model parameterization with the supervision of L. B.). P.C. thanks ONR under Grant No. N00014-17-1-2818 (P. C. wrote the scripts for the model parameterization). C. P. also acknowledges support from the University of Arkansas (C. P. provided suggestions to check whether the model was overfitted). X.L. and Y. Y. acknowledges the support from NSFC (11874207). The numerical simulations were also supported by the Arkansas High Performance Computing Center and the HPC resources of CALMIP (Allocation No. P1229). 
*hz001@uark.edu

†pc011@uark.edu

*laurent@uark.edu

[1] Y. A. Bychkov and E. I. Rashba, JETP Lett. 39, 78 (1984), http://www.jetpletters.ac.ru/ps/1264/article_19121.shtml.

[2] G. Dresselhaus, Phys. Rev. 100, 580 (1955).

[3] R. Moriya, K. Sawano, Y. Hoshi, S. Masubuchi, Y. Shiraki, A. Wild, C. Neumann, G. Abstreiter, D. Bougeard, T. Koga, and T. Machida, Phys. Rev. Lett. 113, 086601 (2014).

[4] H. Nakamura, T. Koga, and T. Kimura, Phys. Rev. Lett. 108, 206601 (2012).

[5] M. Gmitra and J. Fabian, Phys. Rev. B 94, 165202 (2016).

[6] L. L. Tao, T. R. Paudel, A. A. Kovalev, and E. Y. Tsymbal, Phys. Rev. B 95, 245141 (2017).

[7] L. L. Tao and E. Y. Tsymbal, Nat. Commun. 9, 2763 (2018).

[8] D. D. Sante, P. Barone, R. Bertacco, and S. Picozzi, Adv. Mater. 25, 509 (2013).

[9] H. Djani, A. C. Garcia-Castro, W.-Y. Tong, P. Barone, E. Bousquet, S. Picozzi, and P. Ghosez, npj Quantum Mater. 4, 51 (2019).

[10] S. Picozzi, Front. Phys. 2, 10 (2014).

[11] L. G. D. da Silveira, P. Barone, and S. Picozzi, Phys. Rev. B 93, 245159 (2016).

[12] J. Varignon, J. Santamaria, and M. Bibes, Phys. Rev. Lett. 122, 116401 (2019).

[13] E. Plekhanov, P. Barone, D. Di Sante, and S. Picozzi, Phys. Rev. B 90, 161108(R) (2014).

[14] R. Arras, J. Gosteau, H. J. Zhao, C. Paillard, Y. Yang, and L. Bellaiche, Phys. Rev. B 100, 174415 (2019).

[15] C. Autieri, P. Barone, J. Sławińska, and S. Picozzi, Phys. Rev. Mater. 3, 084416 (2019).

[16] A. Stroppa, D. D. Sante, P. Barone, M. Bokdam, G. Kresse, C. Franchini, M.-H. Whangbo, and S. Picozzi, Nat. Commun. 5, 5900 (2014).

[17] K. Ishizaka et al., Nat. Mater. 10, 521 (2011).

[18] S. Bandyopadhyay, A. Paul, and I. Dasgupta, Phys. Rev. B 101, 014109 (2020).

[19] J. D. Koralek, C. P. Weber, J. Orenstein, B. A. Bernevig, S.-C. Zhang, S. Mack, and D. D. Awschalom, Nature (London) 458, 610 (2009).

[20] A. Manchon, H. C. Koo, J. Nitta, S. M. Frolov, and R. A. Duine, Nat. Mater. 14, 871 (2015).

[21] F. Jia, S. Hu, S. Xu, H. Gao, G. Zhao, P. Barone, A. Stroppa, and W. Ren, J. Phys. Chem. Lett. 11, 5177 (2020).

[22] J. Schliemann, J. C. Egues, and D. Loss, Phys. Rev. Lett. 90, 146801 (2003).

[23] J. Schliemann, Rev. Mod. Phys. 89, 011001 (2017).

[24] K. V. Shanavas, Phys. Rev. B 93, 045108 (2016).

[25] W. Lin, L. Li, F. Doğan, C. Li, H. Rotella, X. Yu, B. Zhang, Y. Li, W. S. Lew, S. Wang, W. Prellier, S. J. Pennycook, J. Chen, Z. Zhong, A. Manchon, and T. Wu, Nat. Commun. 10, 3052 (2019).

[26] D. C. Marinescu, Phys. Rev. B 96, 115109 (2017).

[27] J. Schliemann and D. Loss, Phys. Rev. B 71, 085308 (2005).

[28] W. Lin, L. Li, F. Doğan, C. Li, H. Rotella, X. Yu, B. Zhang, Y. Li, W. S. Lew, S. Wang, W. Prellier, S. J. Pennycook, J. Chen, Z. Zhong, A. Manchon, and T. Wu, Nat. Commun. 10, 3052 (2019).

[29] D. Y. Usachov et al., Phys. Rev. Lett. 124, 237202 (2020).

[30] S. Murakami, Science 301, 1348 (2003).
[31] J. Sinova, S. O. Valenzuela, J. Wunderlich, C. H. Back, and T. Jungwirth, Rev. Mod. Phys. 87, 1213 (2015).

[32] M. Willatzen and L.C.L.Y. Voon, The $k p$ Method (Springer, Berlin, Heidelberg, 2009).

[33] See Supplemental Material at http://link.aps.org/ supplemental/10.1103/PhysRevLett.125.216405 for the $\mathbf{k} \cdot \mathbf{p}$ models, crystal and band structures and spin textures of some selected materials, several proposed candidates (for purely cubic spin splittings), and methods used in this Letter, which also includes Refs. [5,6,10,11,18,32,34-61].

[34] Bilbao Crystallographic Server, Point group tables, https:// www.cryst.ehu.es/rep/point.html.

[35] Bilbao Crystallographic Server, Generators and general positions, https://www.cryst.ehu.es/cryst/get_gen.html.

[36] M. El-Batanouny and F. Wooten, Symmetry and Condensed Matter Physics: A Computational Approach (Cambridge University Press, New York, 2008).

[37] Bilbao Crystallographic Server, Irreducible representations of space groups, https://www.cryst.ehu.es/cgi-bin/cryst/ programs/representations.pl?tipogrupo $=\mathrm{spg}$.

[38] https://materialsproject.org/materials/mp-8415.

[39] https://www.materialsproject.org/materials/mp-1572.

[40] Materials project, https://materialsproject.org/.

[41] A. Jain, S. P. Ong, G. Hautier, W. Chen, W. D. Richards, S. Dacek, S. Cholia, D. Gunter, D. Skinner, G. Ceder, and K. A. Persson, APL Mater. 1, 011002 (2013).

[42] G. Kresse and J. Furthmuller, Phys. Rev. B 54, 11169 (1996).

[43] G. Kresse and D. Joubert, Phys. Rev. B 59, 1758 (1999).

[44] P. E. Blochl, Phys. Rev. B 50, 17953 (1994).

[45] G. I. Csonka, J. P. Perdew, A. Ruzsinszky, P. H. T. Philipsen, S. Lebègue, J. Paier, O. A. Vydrov, and J. G. Ángyán, Phys. Rev. B 79, 155107 (2009).

[46] Seek-path, https://www.materialscloud.org/work/tools/ seekpath.

[47] Y. Hinuma, G. Pizzi, Y. Kumagai, F. Oba, and I. Tanaka, Comput. Mater. Sci. 128, 140 (2017).

[48] A. Togo and I. Tanaka, arXiv:1808.01590.

[49] H. T. Stokes and D. M. Hatch, J. Appl. Crystallogr. 38, 237 (2005).

[50] FINDSYM, https://stokes.byu.edu/iso/findsym.php.

[51] pymatgen, https://pymatgen.org/.

[52] S. P. Ong, W. D. Richards, A. Jain, G. Hautier, M. Kocher, S. Cholia, D. Gunter, V. L. Chevrier, K. A. Persson, and G. Ceder, Comput. Mater. Sci. 68, 314 (2013).

[53] K. Momma and F. Izumi, J. Appl. Crystallogr. 44, 1272 (2011).

[54] Mathematica, Version 12.0 (W. R. Inc., Champaign, IL, 2019).

[55] J. D. Hunter, Comput. Sci. Eng. 9, 90 (2007).

[56] H. J. Zhao, P. Chen, C. Paillard, R. Arras, Y.-W. Fang, X. Li, J. Gosteau, Y. Yang, and L. Bellaiche, Phys. Rev. B 102, 041203(R) (2020).

[57] S. L. Altmann and P. Herzig, Point-Group Theory Tables (Oxford Science Publications, Oxford, England, 1994).

[58] M. I. Aroyo, A. Kirov, C. Capillas, J. M. Perez-Mato, and H. Wondratschek, Acta Crystallogr. Sect. A 62, 115 (2006).

[59] M. I. Aroyo, J. M. Perez-Mato, C. Capillas, E. Kroumova, S. Ivantchev, G. Madariaga, A. Kirov, and H. Wondratschek, Z. Kristallogr. 221, 15 (2006). 
[60] S. Vajna, E. Simon, A. Szilva, K. Palotas, B. Ujfalussy, and L. Szunyogh, Phys. Rev. B 85, 075404 (2012).

[61] H. T. Stokes, D. M. Hatch, and B. J. Campbell, FINDSYM, Isotropy Software Suite.

[62] M. Pues and G. Meier, Atomic- and Nanoscale Magnetism (Springer Nature, Switzerland, 2018), Chap. 15.

[63] Y. Iwata, N. Koyano, and I. Shibuya, J. Phys. Soc. Jpn. 35, 1269 (1973).

[64] F. Kubel and H. Vllenkle, Solid State Sci. 2, 193 (2000).

[65] Q. Wu, X. Liu, F. Liang, S. Xu, H. Pi, X. Han, Y. Liu, Z. Lin, and Y. Li, Dalton Trans. 48, 13529 (2019).

[66] Manuel Bibes (personal communication).
[67] D. Xiao, G.-B. Liu, W. Feng, X. Xu, and W. Yao, Phys. Rev. Lett. 108, 196802 (2012).

[68] X. Zhang, Q. Liu, J.-W. Luo, A. J. Freeman, and A. Zunger, Nat. Phys. 10, 387 (2014).

[69] G. Wang, A. Chernikov, M. M. Glazov, T. F. Heinz, X. Marie, T. Amand, and B. Urbaszek, Rev. Mod. Phys. 90, 021001 (2018).

[70] K. S. Novoselov, A. Mishchenko, A. Carvalho, and A. H. C. Neto, Science 353, aac9439 (2016).

[71] Q. H. Wang, K. Kalantar-Zadeh, A. Kis, J. N. Coleman, and M. S. Strano, Nat. Nanotechnol. 7, 699 (2012).

[72] USPEX, https://uspex-team.org/en/uspex/overview.

[73] CALYPSO, http://www.calypso.cn/. 